\title{
Fibroblast Growth Factor 21 and Metabolic Dysfunction in Women with a Prior Glucose-Intolerant Pregnancy
}

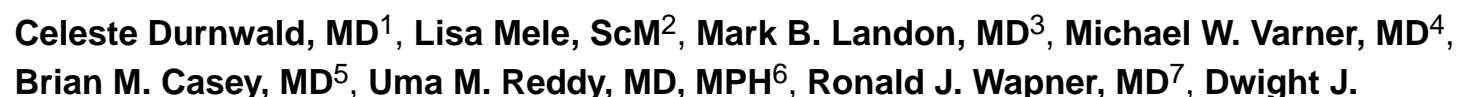
Rouse, MD ${ }^{8}$, Alan T. N. Tita, MD, PhD $^{9}$, John M. Thorp Jr., MD ${ }^{10}$, Edward K. Chien, MD, MBA $^{11}$, George R. Saade, MD ${ }^{12}$, Alan M. Peaceman, MD ${ }^{13}$, Sean C. Blackwell, MD ${ }^{14}$, Eunice Kennedy Shriver National Institute of Child Health Human Development (NICHD) Maternal Fetal Medicine Units (MFMU) Network ${ }^{*}$

${ }^{1}$ Department of Obstetrics and Gynecology, University of Pennsylvania, Philadelphia, Pennsylvania

${ }^{2}$ George Washington University Biostatistics Center, Washington, District of Columbia ${ }^{3}$ Department of Obstetrics and Gynecology, The Ohio State University, Columbus, Ohio ${ }^{4}$ Department of Obstetrics and Gynecology, University of Utah Health Sciences Center, Salt Lake City, Utah

${ }^{5}$ Department of Obstetrics and Gynecology, University of Texas Southwestern Medical Center, Dallas, Texas

${ }^{6}$ Eunice Kennedy Shriver National Institute of Child Health and Human Development (U.M.R.), Bethesda, MD

${ }^{7}$ Department of Obstetrics and Gynecology, Columbia University, New York, New York ${ }^{8}$ Department of Obstetrics and Gynecology, Brown University, Providence, Rhode Island ${ }^{9}$ Department of Obstetrics and Gynecology, University of Alabama at Birmingham, Birmingham, Alabama

${ }^{10}$ Department of Obstetrics and Gynecology, University of North Carolina, Chapel Hill, North Carolina

${ }^{11}$ Department of Obstetrics and Gynecology, MetroHealth Medical Center - Case Western Reserve University, Cleveland, Ohio

${ }^{12}$ Department of Obstetrics and Gynecology, University of Texas Medical Branch, Galveston, Texas

${ }^{13}$ Department of Obstetrics and Gynecology, Northwestern University, Chicago, Illinois

\footnotetext{
*See Supplementary Material (available in the online version) for list of other members of the NICHD MFMU Network, Bethesda, MD.

Address for correspondence Celeste Durnwald, MD, University of Pennsylvania, 3400 Spruce Street, 2 Silverstein, Philadelphia, PA 19104 ( celeste.durnwald@uphs.upenn.edu).

Conflict of Interest

None declared.
} 
${ }^{14}$ Department of Obstetrics and Gynecology, University of Texas Health Science Center at Houston-Children's Memorial Hermann Hospital, Houston, Texas

\section{Abstract}

Objective-We sought to determine if there is an association between fibroblast growth factor 21 (FGF21) levels and a history of gestational diabetes mellitus (GDM) in women with and without metabolic dysfunction, defined as a diagnosis of metabolic syndrome or type 2 diabetes (T2DM), 5 to 10 years following participation in a multiple cohort GDM study.

Study Design-At 5 to 10 years after index pregnancy, women underwent a follow-up visit and were categorized as having no metabolic syndrome, metabolic syndrome, or T2DM. FGF21 levels were compared between women who did and did not have a history of GDM using multivariable linear regression.

Results-Among 1,889 women, 950 underwent follow-up and 796 had plasma samples analyzed (413 GDM and 383 non-GDM). Total 30.7\% of women had been diagnosed with T2DM or metabolic syndrome. Overall, there was no difference in median FGF21 levels in $\mathrm{pg} / \mathrm{mL}$ between the prior GDM and non-GDM groups $(p=0.12)$, and the lack of association was observed across all three metabolic categories at follow-up $(p$ for interaction $=0.70$ ).

Conclusion-There was no association between FGF21 levels and prior history of mild GDM in women with and without metabolic dysfunction 5 to 10 years after the index pregnancy (ClinicalTrials.gov number, NCT00069576, original trial).

\section{Keywords}

fibroblast growth factor; gestational diabetes; glucose intolerance; metabolic syndrome

Women with a history of gestational diabetes mellitus (GDM) are at increased risk for the development of type 2 diabetes mellitus (T2DM) and the metabolic syndrome. Approximately one half of these women develop diabetes in the first 5 to 10 years after the index pregnancy. ${ }^{1}$ However, the rate of conversion to T2DM can vary widely, with ranges reported from 2.6 to $70 \%$ over a period from 6 weeks to 26 years from the index pregnancy. ${ }^{2}$ A history of GDM is also a risk factor for cardiovascular disease, such as dyslipidemia and coronary artery disease later in life. ${ }^{3,4}$ Fibroblast growth factor 21 (FGF21) is a recognized metabolic regulator of glucose and lipid metabolism. Elevated levels of FGF21 have been identified in individuals with T2DM, atherosclerosis, coronary artery disease, and acute myocardial infarction. FGF21 holds promise as a potential biomarker not only for the development of T2DM, but also for the vascular complications associated with diabetes. ${ }^{5}$ Because the pathophysiology of GDM and T2DM is similar, both involving insulin resistance and impaired $\beta$ cell function - the relationship between FGF2 1 and GDM - has been explored. ${ }^{6}$ Small studies have shown that second and third trimester FGF21 levels are higher in GDM women compared with women with normal glucose tolerance. ${ }^{7-9}$ Although the exact mechanism of elevations in FGF21 is unknown, there is speculation that the upregulation of FGF21 may be a compensatory response in an attempt to improve glucose metabolism when insulin resistance is high. In two 5-year prospective studies, elevations in FGF21 have also been shown to predict the development of metabolic 
syndrome and T2DM. ${ }^{10,11}$ Given that elevations in FGF21 have been shown to predict varying degrees of glucose intolerance, it is plausible that FGF21 could be used to identify a subset of women with a history of prior GDM who may be at risk for the development of metabolic disorders long term. Therefore, our objective was to determine if there is an association between FGF21 levels and a prior GDM pregnancy in women with and without metabolic dysfunction (a diagnosis of metabolic syndrome or T2DM) 5 to 10 years, including metabolic syndrome and T2DM, following participation in a multiple cohort GDM study.

\section{Study Design}

This is a secondary analysis of women enrolled during pregnancy with and without mild GDM in a multicenter trial in the Eunice Kennedy Shriver National Institute of Child Health and Human Development Maternal Fetal Medicine Units Network GDM follow-up study who were evaluated 5 to 10 years after their index pregnancy. Details regarding the original trial have been previously described. ${ }^{12}$ Mild GDM was defined with a 3-hour, 100 -g oral glucose tolerance test (OGTT) with a fasting glucose of less than $95 \mathrm{mg} / \mathrm{dL}$, but at least two of the 1-, 2-, or 3-hour determinations that exceeded established thresholds of 1 hour $>180 \mathrm{mg} / \mathrm{dL}, 2$ hours $>155 \mathrm{mg} / \mathrm{dL}$, and 3 hours $>140-\mathrm{mg} / \mathrm{dL}$ satisfying the criteria for a positive result. Non-GDM women were defined as women with a 50-g, 1-hour screening test between 135 and $200 \mathrm{mg} / \mathrm{dL}$ who underwent a 100-g, 3-hour OGTT with normal results and matched by race/ethnicity and body mass index (BMI) to the women with mild GDM. Women did not have a history of pregestational or GDM, renal or cardiovascular disease, or chronic hypertension at the time of enrollment in the original study. Once GDM women were consented, women were randomly assigned to receive either formal nutritional counseling and diet therapy, along with insulin if needed (treated) or usual prenatal care (untreated). The parent mild GDM Trial and the follow-up study were approved by the institutional review board of all participating centers. Thefollow-up study has been previously described. ${ }^{13}$ At the 5 to 10 year follow-up, women underwent anthropometric and blood pressure measurements and completed a 75-g, 2-hour OGTT. Additional fasting blood was obtained and saved for future analysis. Womenwere then categorized as having no metabolic syndrome (0-2 components of the formal diagnosis of metabolic syndrome), metabolic syndrome or T2DM at follow-up. Metabolic syndrome was defined per the American Heart Association and National Heart Lung and Blood Institute Scientific Statement including three or greater of the following: waist circumference $>88$ $\mathrm{cm}$, triglyceride level $\geq 150 \mathrm{mg} / \mathrm{dL}$, high-density lipoprotein $<50 \mathrm{mg} / \mathrm{dL}$, blood pressure $\geq 130 / 85 \mathrm{~mm} \mathrm{Hg}$, or fasting glucose $\geq 100 \mathrm{mg} / \mathrm{dL} .{ }^{14}$ Diabetes was defined as a fasting glucose $\geq 126 \mathrm{mg} / \mathrm{dL}$ and/or 2-hour glucose $\geq 200$ - $\mathrm{mg} / \mathrm{dL}$ after a 75 -g load or if a woman was currently undergoing treatment for a prior diagnosis of T2DM. ${ }^{15}$ Women with no metabolic syndrome had 0 to 2 components of the criteria for the metabolic syndrome defined above.

Women were excluded from the current study if they did not fast for at least 6 hours prior to the fasting blood draw or if they had incomplete study visit data (no waist circumference measurement, no fasting lipid profile, no BP measurement, or no 2-hour OGTT). Women 
with no fasting aliquot sample available or if there was inadequate amount of protein to run the FGF21 assay once the sample was thawed were also excluded.

FGF21 levels were assayed using the Quantikine ELISA Human FGF21 Immunoassay kit (R\&D Systems, Minneapolis, MN). The sensitivity of the Human FGF-21 Quantikine Elisa assay range from 1.61 to $8.69 \mathrm{pg} / \mathrm{mL}$. The mean of the minimum detectable dose is 4.67 $\mathrm{pg} / \mathrm{mL}$. Three samples of known concentration were tested 20 times on one plate to assess intra-assay precision. Three samples of known concentration were tested in 40 separate assays to assess inter-assay precision. Assays were performed by at least three technicians using two lots of components. To determine if the detection of FGF-21 protein was in the standard curve range, undiluted samples and a series of dilutions from three random plasma samples from women enrolled in a different study were run. Undiluted sample results were in the standard curve range. A human FGF-21 standard curve was generated for each plate. Samples were thawed on ice and assayed in duplicate following assay procedure. Optical density was determined using a microplate reader set to $450 \mathrm{~nm}$. FGF21 levels were analyzed as a continuous variable.

Baseline characteristics were compared between women who did and did not have a history of GDM. The Chi-square test and, in the case of small numbers, Fisher's exact test were used to examine categorical variables and the Wilcoxon's rank sum test was used to examine continuous variables. Univariate analysis comparing FGF21 levels between two groups (e.g., GDM and non-GDM groups) was undertaken using the Wilcoxon's rank sum test and for more than two groups using the Kruskal-Wallis test (e.g., GDM treated, untreated, and nonGDM groups). Multivariable analysis was performed using multivariable linear regression models adjusting for possible confounders and included maternal age, maternal BMI, and time interval in years between the original trial and the follow-up visit. Since the distribution of FGF21 values showed a right skewed distribution, FGF21 values were log-transformed in the regression analysis to approximate normality. Interaction between the original diagnosis of GDM and current metabolic status on FGF21 levels was also examined in regression models.

Additional regression models were run, separately, based on current metabolic status, categorized as no metabolic dysfunction, metabolic syndrome, and T2DM. Imputation for missing data was not performed. Nominal $p$ values of less than 0.05 were considered to indicate statistical significance. No adjustments were made for multiple comparisons. Statistical analysis was performed using SAS 9.4 statistical software (SAS Institute Inc., Cary, NC).

\section{Results}

Among 1,889 women enrolled in the original trial, 950 (50\%) completed a study visit for the GDM follow-up study. Of these women, 796 (83.8\%) had plasma samples available for analysis. A total of 154 women were excluded for the following: 62 currently pregnant, 62 no fasting blood draw/missing metabolic syndrome data, 29 no sample, and 1 sample had inadequate amount of protein to run the FGF21 assay. Of the women included, 413 were diagnosed with GDM during the index pregnancy, 220 of whom underwent treatment 
for GDM, and 193 had untreated GDM. Additionally, there were 383 non-GDM women (elevated 1-hour glucose challenge test [GCT], normal 3-hour OGTT). The mean duration from study index pregnancy delivery to follow-up was $6.9 \pm 1.4$ years.

Maternal clinical characteristics of women at follow-up study visit based on mild GDM status in the original trial are listed in Table 1. GDM and non-GDM women were similar except for maternal age, insulin therapy during the index pregnancy, and number of GDM pregnancies since the index pregnancy, all significantly higher in GDM women. At the time of the follow-up study visit, 200 women (58 prior treated GDM, 56 untreated GDM, and 86 non-GDM) were diagnosed with metabolic syndrome, 44 had T2DM (18 prior treated GDM, 14 untreated GDM, and 12 non-GDM) and 552 women (144 treated GDM, 123 untreated GDM, and 285 non-GDM) did not meet criteria for metabolic syndrome. Of the 552 women without metabolic syndrome, 245 had two criteria of the metabolic syndrome, 173 had one criterion, and 134 had none.

Overall, in univariate analysis, there was no difference in median FGF21 levelsinpg/mL between the prior GDM and non-GDM groups (169.3 [interquartile range $\{\mathrm{QR}\}$ : 98.4261.6] vs. 153.4 [IQR: 84.4-241.1], respectively, $p=0.08$ [Wilcoxon's test]). In adjusted analyses utilizing linear regression modeling, there was also no association observed between prior GDM and non-GDM groups and log-transformed FGF21 values, after adjusting for maternal age, maternal BMI, and time interval between the original trial and the follow-up visit $(p=0.12$ ). Additionally, there was no significant interaction between GDM group and metabolic category at follow-up with FGF21 levels $(p$ for interaction $=$ 0.70; Table 2). Similarly, there were no significant differences in FGF21 levels when the GDM group was analyzed according to whether or not their prior GDM was treated ( Table 3).

In a cross-sectional analysis, median FGF21 levels were significantly higher in women with a diagnosis of T2DM (235.8 [IQR: 155.8-411.4]) and metabolic syndrome (202.3 [IQR: 129.0-308.6]) compared with no metabolic syndrome (140.3 [IQR: 80.1-226.4]), $(p<0.001$ [Kruskal-Wallis test]).

Of the 552 women without a formal diagnosis of metabolic syndrome, there were significant differences in FGF21 levels based on the number of components of metabolic syndrome they experienced from no components to two components ( $p<0.001$ [Kruskal-Wallis test]).

\section{Conclusion}

In a follow-up of a multiple cohort GDM study, we did not find an association between FGF21 levels measured 5 to 10 years postdelivery and a history of a GDM pregnancy.

We were able to demonstrate that FGF21 levels were significantly different among the groups based on their current metabolic status. Specifically, women with a diagnosis of T2DM, either prior to the study visit or at the time of the study visit had the highest levels of FGF21 and women with no metabolic syndrome having the lowest FGF21 levels. Women with a diagnosis of metabolic syndrome, as defined by the AHA/NHLBI, had higher FGF21 levels compared with women who were not diagnosed with metabolic syndrome, but lower 
levels than those with T2DM. Our findings are consistent with prior reports demonstrating that FGF21 levels are elevated in individuals with both metabolic syndrome and T2DM. ${ }^{7}$ We were unable to demonstrate additional influence of a history of GDM in prior pregnancy on FGF21 levels.

Fibroblast growth factor is an important metabolic regulator with a significant effect on glucose homeostasis. Increased levels have been identified in individuals with varying degrees of insulin resistance including obesity and T2DM. ${ }^{16,17}$ In the GDM pregnancy, insulin resistance is mainly related to circulating placental hormones, although baseline maternal obesity may also play a role. Elevations in FGF21 have also been reported in women with GDM during pregnancy compared with women with normal glucose tolerance. ${ }^{7,8}$ In two studies of predominantly Chinese women, circulating FGF21 levels were higher among GDM women compared with those with normal glucose tolerance. ${ }^{7,8}$ Similarly, in a small group of Caucasian women, the association between elevations in FGF21 levels in those with GDM remained even when matched for maternal age and BMI. ${ }^{9}$ These findings were also confirmed in a larger population of women of EuropeanMediterranean descent. ${ }^{18}$ Whether elevations in FGF21 persist after delivery in GDM women known to be at increased risk for long-term metabolic and vascular dysfunction has not been well studied.

As clinicians, we are in search of biomarkers that may aid in identifying women at risk for disease as a point of intervention that may alter progression to that particular disease. GDM is no exception, especially when follow-up rates of postpartum screening are low. ${ }^{19,20}$ Women with a history of GDM possess higher levels of inflammation which may result in changes in inflammatory and metabolic markers years prior to a formal diagnosis of T2DM. ${ }^{21}$ Therefore, identifying a subset of prior GDM women who have not yet developed metabolic syndrome or T2DM in the 5 to 8 years following the index pregnancy, but who are still at risk for metabolic disorders long term could provide an intermediate window for interventions such as pharmacologic therapy and lifestyle interventions. Previous studies have focused on adiponectin and high sensitivity $\mathrm{C}$-reactive protein as potential biomarkers in GDM women and risk of progression to T2DM with mixed results. ${ }^{22}$ In non-GDM studies, FGF21 has been shown to be superior to adiponectin in predicting future diabetes. ${ }^{23}$ Elevated levels of FGF21 prior to a formal diagnosis of T2DM have been confirmed in mixed populations. In a 5-year prospective study of Chinese subjects, plasma FGF21 levels progressively increased through the continuum of glucose tolerance from normal glucose tolerance to diabetes. ${ }^{10}$ Similarly, in a Caucasian population of men and women, FGF21 levels drawn 5 years earlier were independently associated with the development of both T2DM and metabolic syndrome, with a twofold increased risk of metabolic dysfunction compared with normal glucose tolerant individuals. ${ }^{11}$ In the Fenofibrate Intervention and Event Lowering in Diabetes (FIELD) study, elevations in FGF21 were also associated with a higher risk of cardiovascular events in participants with T2DM. ${ }^{24}$ Higher levels of FGF21 were associated with both the baseline presence of microvascular complications (nephropathy, retinopathy, and neuropathy), and also with development of microvascular complications in T2DM without preexisting vascular complications in a sub-analysis of the FIELD study. ${ }^{5}$ 
Since women with GDM are at increased risk for metabolic syndrome and T2DM later in life, FGF21 may be an ideal biomarker to identify women who are predisposed to impaired glucose tolerance but whose glucose levels are below current diagnostic thresholds for either metabolic syndrome or T2DM.2 Animal models show promise for using FGF21 as a potential therapeutic target to increase insulin sensitivity and decrease blood glucose concentrations. ${ }^{25,26}$ If these results can be replicated in human studies, clinical development of FGF21 therapies could be used in the treatment of obesity and T2DM. Despite prior findings, we were unable to identify an independent association between FGF21 levels 5 and 10 years after exposure to a glucose intolerant pregnancy and a prior diagnosis of GDM. The lack of association was seen in all three metabolic categories, including the $69.3 \%$ of women who still had no evidence of metabolic syndrome.

Our study has several limitations. Women with normal 1-hour GCT were not included in the GDM follow-up study. Therefore, we were unable to evaluate whether differences in FGF21 levels might have been detected between women with mild GDM and women with normal 1-hour GCT results. In our study, women had a mild phenotype of GDM with a normal fasting glucose on the 3-hour OGTT and only 39 women in the original trial required insulin therapy to achieve adequate glucose control. Although we did not find an association between FGF21 levels and women with a history of mild GDM, it is plausible that an association may exist between FGF21 levels and women with a history of more severe phenotypes of GDM.

In summary, we were unable to demonstrate an association between FGF21 levels and prior history of a GDM pregnancy in women with and without metabolic syndrome or T2DM 5 to 10 years after the index pregnancy. Our results are consistent with prior studies showing a strong association between FGF21 levels and current metabolic status. Research efforts should continue to focus on approaches to improve intermediate and long-term follow-up of women with a history of GDM to identify points of intervention to prevent or delay progression to long term metabolic and vascular dysfunction in this at risk group of women.

\section{Supplementary Material}

Refer to Web version on PubMed Central for supplementary material.

\section{Acknowledgments}

The authors thank Francee Johnson, RN, BSN, and Lisa Moseley, RN, for protocol development and coordination between clinical research centers; Lindsey Doherty, MS, for protocol and data management; and Elizabeth Thom, $\mathrm{PhD}$, Madeline M. Rice, $\mathrm{PhD}$, and Catherine Y. Spong, MD, for protocol development and oversight.

Funding

This study was supported by grants from the Eunice Kennedy Shriver National Institute of Child Health and Human Development (grant numbers: HD27915, HD36801, HD34208, HD34116, HD40485, HD40500, HD27869, HD40560, HD40544, HD53097, HD40512, and HD40545) and the National Institutes of Health's National Center for Advancing Translational Sciences (grant numbers: UL1TR001070 and UL1TR000439). Comments and views of the authors do not necessarily represent views of the National Institutes of Health.

This study was presented in part at the 2018 Annual Scientific Meeting of the Society for Maternal-Fetal Medicine (January 29 to February 3, 2017), Dallas, TX. 


\section{References}

1. Bellamy L, Casas JP, Hingorani AD, Williams D. Type 2 diabetes mellitus after gestational diabetes: a systematic review and meta-analysis. Lancet 2009;373(9677):1773-1779 [PubMed: 19465232]

2. Kim C, Newton KM, Knopp RH. Gestational diabetes and the incidence of type 2 diabetes: a systematic review. Diabetes Care 2002;25(10):1862-1868 [PubMed: 12351492]

3. Heitritter SM, Solomon CG, Mitchell GF, Skali-Ounis N, Seely EW. Subclinical inflammation and vascular dysfunction in women with previous gestational diabetes mellitus. J Clin Endocrinol Metab 2005;90(07):3983-3988 [PubMed: 15840749]

4. Li JW, He SY, Liu P, Luo L, Zhao L, Xiao YB. Association of gestational diabetes mellitus (GDM) with subclinical atherosclerosis: a systemic review and meta-analysis. BMC Cardiovasc Disord 2014;14:132 [PubMed: 25266849]

5. Ong KL, Januszewski AS, O'Connell R, et al. ; FIELD study investigators. Relationship of fibroblast growth factor 21 with baseline and new on-study microvascular disease in the Fenofibrate Intervention and Event Lowering in Diabetes study. Diabetologia 2015;58(09):20352044 [PubMed: 26055067]

6. Yuan D, Wu BJ, Henry A, Rye KA, Ong KL. Role of fibroblast growth factor 21 in gestational diabetes mellitus: A mini-review. Clin Endocrinol (Oxf) 2019;90(01):47-55

7. Wang D, Zhu W, Li J, An C, Wang Z. Serum concentrations of fibroblast growth factors 19 and 21 in women with gestational diabetes mellitus: association with insulin resistance, adiponectin, and polycystic ovary syndrome history. PLoS One 2013;8(11):e81190 [PubMed: 24260557]

8. Li SM, Wang WF, Zhou LH, et al. Fibroblast growth factor 21 expressions in white blood cells and sera of patients with gestational diabetes mellitus during gestation and postpartum. Endocrine 2015;48(02):519-527 [PubMed: 24895044]

9. Tan BK, Sivakumar K, Bari MF, Vatish M, Randeva HS. Lower cerebrospinal fluid/plasma fibroblast growth factor 21 (FGF21) ratios and placental FGF21 production in gestational diabetes. PLoS One 2013;8(06):e65254 [PubMed: 23755203]

10. Chen C, Cheung BM, Tso AW, et al. High plasma level of fibroblast growth factor 21 is an Independent predictor of type 2 diabetes: a 5.4-year population-based prospective study in Chinese subjects. Diabetes Care 2011;34(09):2113-2115 [PubMed: 21750278]

11. Bobbert T, Schwarz F, Fischer-Rosinsky A, et al. Fibroblast growth factor 21 predicts the metabolic syndrome and type 2 diabetes in Caucasians. Diabetes Care 2013;36(01):145-149 [PubMed: 22933429]

12. Landon MB, Spong CY, Thom E, et al. ; Eunice Kennedy Shriver National Institute of Child Health and Human Development Maternal-Fetal Medicine Units Network. A multicenter, randomized trial of treatment for mild gestational diabetes. N Engl J Med 2009;361(14):13391348 [PubMed: 19797280]

13. Landon MB, Rice MM, Varner MW, et al. ; Eunice Kennedy Shriver National Institute of Child Health and Human Development Maternal-Fetal Medicine Units (MFMU) Network. Mild gestational diabetes mellitus and long-term child health. Diabetes Care 2015;38(03):445-452 [PubMed: 25414152]

14. Grundy SM, Cleeman JI, Daniels SR, et al. ; American Heart Association; National Heart, Lung, and Blood Institute. Diagnosis and management of the metabolic syndrome: an American Heart Association/National Heart, Lung, and Blood Institute Scientific Statement. Circulation 2005;112(17):2735-2752 [PubMed: 16157765]

15. American Diabetes Association. 2. Classification and Diagnosis of Diabetes: Standards of Medical Care in Diabetes-2018. Diabetes Care 2018;41(Suppl 1):S13-S27 [PubMed: 29222373]

16. Mraz M, Bartlova M, Lacinova Z, et al. Serum concentrations and tissue expression of a novel endocrine regulator fibroblast growth factor- 21 in patients with type 2 diabetes and obesity. Clin Endocrinol (Oxf) 2009;71(03):369-375 [PubMed: 19702724]

17. Zhang F, Yu L, Lin X, et al. Minireview: roles of fibroblast growth factors 19 and 21 in metabolic regulation and chronic diseases. Mol Endocrinol 2015;29(10):1400-1413 [PubMed: 26308386]

18. Megia A, Gil-Lluis P, Näf S, et al. Cord blood FGF21 in gestational diabetes and its relationship with postnatal growth. Acta Diabetol 2015;52(04):693-700 [PubMed: 25604041] 
19. Gabbe SG, Landon MB, Warren-Boulton E, Fradkin J. Promoting health after gestational diabetes: a National Diabetes Education Program call to action. Obstet Gynecol 2012;119(01): 171-176 [PubMed: 22183225]

20. Nielsen KK, Kapur A, Damm P, de Courten M, Bygbjerg IC. From screening to postpartum follow-up - the determinants and barriers for gestational diabetes mellitus (GDM) services, a systematic review. BMC Pregnancy Childbirth 2014;14:41 [PubMed: 24450389]

21. Ozuguz U, Isik S, Berker D, et al. Gestational diabetes and subclinical inflammation: evaluation of first year postpartum outcomes. Diabetes Res Clin Pract 2011;94(03):426-433 [PubMed: 21917349]

22. Durnwald CP, Downes K, Leite R, Elovitz M, Parry S. Predicting persistent impaired glucose tolerance in patients with gestational diabetes: The role of high sensitivity CRP and adiponectin. Diabetes Metab Res Rev 2018;34(02):34

23. Woo YC, Lee CH, Fong CH, et al. Serum fibroblast growth factor 21 is a superior biomarker to other adipokines in predicting incident diabetes. Clin Endocrinol (Oxf) 2017;86(01):37-43 [PubMed: 27611701]

24. Ong KL, Januszewski AS, O'Connell R, et al. The relationship of fibroblast growth factor 21 with cardiovascular outcome events in the Fenofibrate Intervention and Event Lowering in Diabetes study. Diabetologia 2015;58(03):464-473 [PubMed: 25425220]

25. Xu J, Stanislaus S, Chinookoswong N, et al. Acute glucose-lowering and insulin-sensitizing action of FGF21 in insulin-resistant mouse models-association with liver and adipose tissue effects. Am J Physiol Endocrinol Metab 2009;297(05):E1105-E1114 [PubMed: 19706786]

26. Xu J, Lloyd DJ, Hale C, et al. Fibroblast growth factor 21 reverses hepatic steatosis, increases energy expenditure, and improves insulin sensitivity in diet-induced obese mice. Diabetes 2009;58 (01):250-259 [PubMed: 18840786] 


\section{Table 1}

Clinical characteristics of the women at study follow-up based on mild gestational diabetes mellitus status in the original trial

\begin{tabular}{|c|c|c|}
\hline & Prior GDM $n=413$ & Prior non-GDM $n=383$ \\
\hline Maternal age (y) & $36.4 \pm 5.4$ & $34.6 \pm 5.6$ \\
\hline \multicolumn{3}{|l|}{ BMI $\left(\mathrm{kg} / \mathrm{m}^{2}\right)$} \\
\hline Normal & $97(23.5)$ & $91(23.8)$ \\
\hline Overweight & $150(36.3)$ & $138(36.0)$ \\
\hline Obese & $166(40.2)$ & $154(40.2)$ \\
\hline \multicolumn{3}{|l|}{ Race/ethnicity } \\
\hline White & $131(31.7)$ & $116(30.3)$ \\
\hline Black & $39(9.4)$ & $42(11.0)$ \\
\hline Hispanic & $231(55.9)$ & $217(56.7)$ \\
\hline Other & $12(2.9)$ & $8(2.1)$ \\
\hline \multicolumn{3}{|l|}{ Insurance status } \\
\hline Private & $142(34.4)$ & $121(31.6)$ \\
\hline Government & $130(31.5)$ & $124(32.4)$ \\
\hline Self pay & $141(34.1)$ & $138(36.0)$ \\
\hline Time since index GDM pregnancy $(y)$ & $7.0 \pm 1.4$ & $6.8 \pm 1.4$ \\
\hline Insulin therapy in index pregnancy, $n(\%)$ & $18(4.4)$ & 0 \\
\hline Preeclampsia in index pregnancy, $n(\%)$ & $15(3.6)$ & $15(3.9)$ \\
\hline Subsequent pregnancy since GDM trial & $205(49.6)$ & $210(54.8)$ \\
\hline \multicolumn{3}{|l|}{ Pregnancies with GDM since index pregnancy } \\
\hline None & $351(85.0)$ & $355(92.7)$ \\
\hline 1 & $54(13.1)$ & $25(6.5)$ \\
\hline $2+$ & $8(1.9)$ & $3(0.8)$ \\
\hline Years since last pregnancy $(\mathrm{y})$ & $3.0 \pm 2.1$ & $2.9 \pm 2.0$ \\
\hline Weight gain since GDM index pregnancy $(\mathrm{kg})$ & $7.3 \pm 10.0$ & $7.2 \pm 9.8$ \\
\hline Chronic hypertension, $n(\%)$ & $27(7.5)$ & $24(7.4)$ \\
\hline Hypercholesterolemia, $n(\%)$ & $51(21.2)$ & $38(19.0)$ \\
\hline
\end{tabular}

Abbreviations: BMI, Body mass index; GDM, gestational diabetes mellitus. Note: Data expressed as $n(\%)$ or mean \pm standard deviation. 


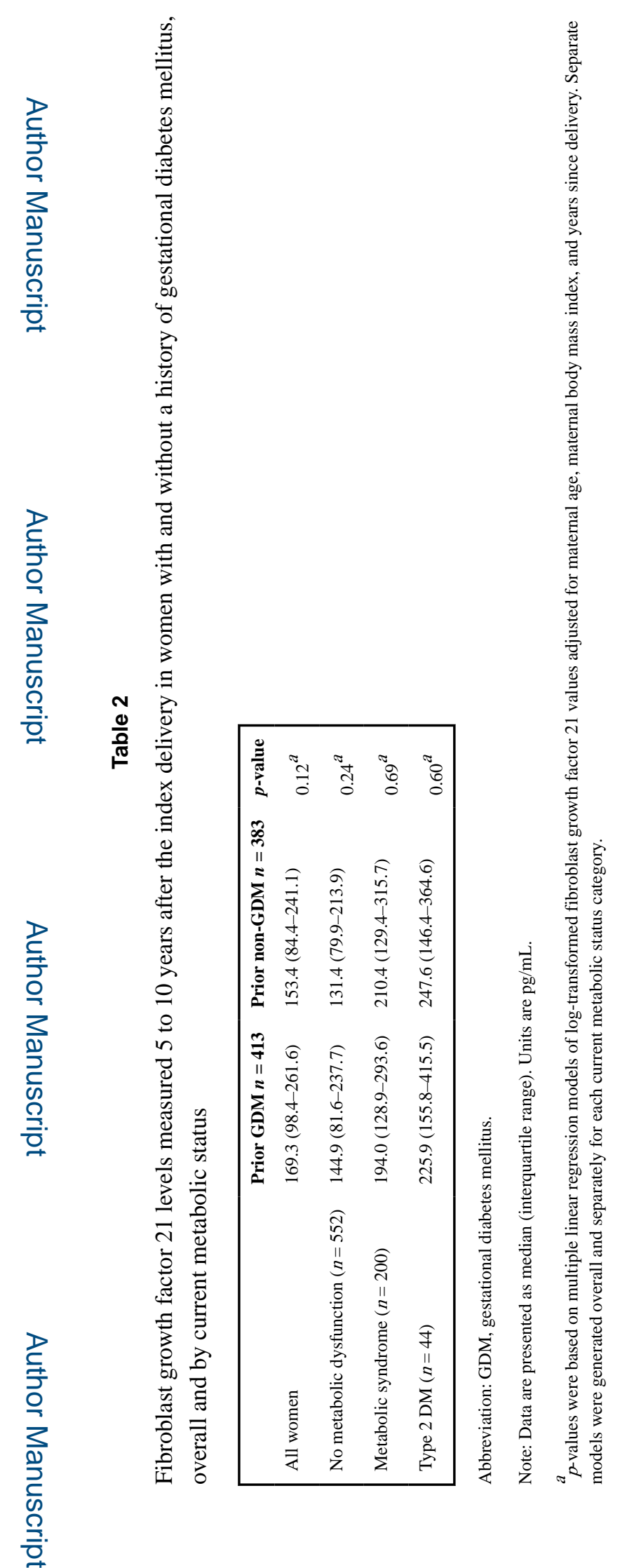

Am J Perinatol. Author manuscript; available in PMC 2021 November 04. 

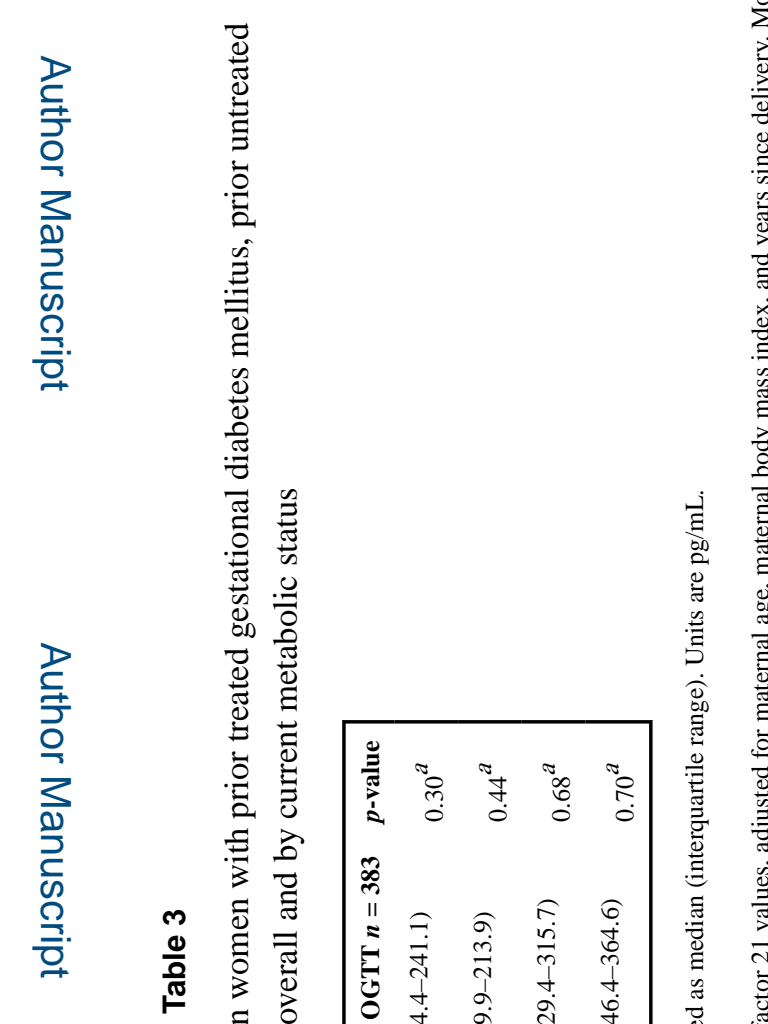

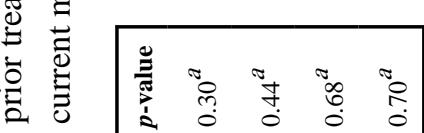

고

范

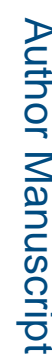

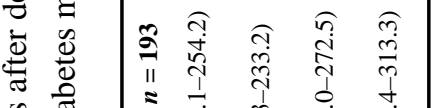

음

苛

응

它莺

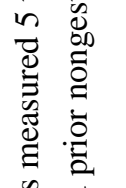

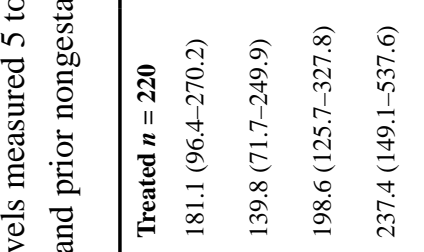

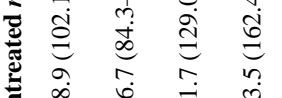

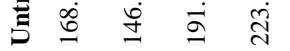

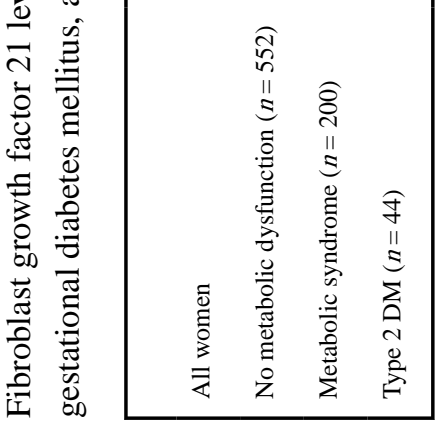

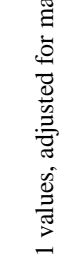

竞

एँ 